\title{
Lumen-apposing metal stent through the meshes of duodenal metal stents for palliation of malignant jaundice
}

\section{(ㄷ)(1) $\odot$}

\section{Authors}

Benedetto Mangiavillano ${ }^{1,2}$, Rastislav Kunda ${ }^{3,4}$, Carlos Robles-Medranda ${ }^{5}$, Roberto Oleas ${ }^{5}$, Andrea Anderloni ${ }^{6}$, Adrien Sportes ${ }^{7}$, Carlo Fabbri ${ }^{8}$, Cecilia Binda ${ }^{8}$, Francesco Auriemma ${ }^{1}$, Leonardo H. Eusebi ${ }^{9}$, Leonardo Frazzoni ${ }^{9}$, Lorenzo Fuccio $^{9}$, Matteo Colombo ${ }^{6}$, Alessandro Fugazza ${ }^{6}$, Mario Bianchetti ${ }^{1}$, Alessandro Repici ${ }^{2,6}$

Institutions

1 Gastrointestinal Endoscopy Unit - Humanitas Mater Domini - Castellanza (VA), Italy

2 Humanitas University, Pieve Emanuele (MI), Italy

3 Department of Surgery, Department of Gastroenterology and Hepatology, and Department of Advanced Interventional Endoscopy, Universitair Ziekenhuis Brussel, Vrije Universiteit Brussel, Brussels, Belgium

4 Department of Surgical Gastroenterology, Aarhus University Hospital, Aarhus, Denmark

5 Instituto Ecuatoriano de Enfermedades Digestivas (IECED), Guayaquil, Ecuador

6 Endoscopy Unit, Istituto Clinico Humanitas Research Hospital, Milan

7 Digestive Endoscopy, Institut Arnault TZANCK Gastroenterology Unit, Saint-Laurent-du-Var, France

8 Digestive Endoscopy Unit, Endoscopy Unit, MorgagniPietrantoni Hospital, Forlì, Italy

9 Endoscopy Unit, Department of Medical and Surgical Sciences, Sant'Orsola University Hospital, Bologna, Italy

\section{Bibliography}

Endoscopy International Open 2021; 09: E324-E330

DOI 10.1055/a-1333-1053

ISSN 2364-3722

(c) 2021. The Author(s).

This is an open access article published by Thieme under the terms of the Creative Commons Attribution-NonDerivative-NonCommercial License, permitting copying and reproduction so long as the original work is given appropriate credit. Contents may not be used for commecial purposes, or adapted, remixed, transformed or built upon. (https://creativecommons.org/licenses/by-nc-nd/4.0/)

Georg Thieme Verlag KG, Rüdigerstraße 14,

70469 Stuttgart, Germany
Corresponding author

Benedetto Mangiavillano, MD, Gastrointestinal Endoscopy

Unit, Humanitas - Mater Domini, Via Gerenzano n.2,

21053-Castellanza (VA)., Italy

Fax: +00390331476210

bennymangiavillano@gmail.com

\section{ABSTRACT}

Background and study aims Endoscopic retrograde cholangiopancreatography (ERCP) is the gold standard procedure for malignant jaundice palliation; however, it can be challenging when a duodenal self-expandable metal stent (SEMS) is already in place.

Patients and methods The primary aim of our study was to evaluate the technical feasibility of the placement of a lumen apposing metal stent (LAMS) through the mesh (TTM) of duodenal stents. The secondary aims were to evaluate clinical outcomes and adverse events (AEs) related to the procedures.

Results Data from 23 patients ( $11 \mathrm{~F}$ and $12 \mathrm{M}$; mean age: $69.5 \pm 11$ years old) were collected. In 17 patients $(73.9 \%)$ TTM LAMS placement was performed as first intention, while in six patients $(26.1 \%)$ it was performed after a failed ERCP. Thirteen patients (56.5\%) underwent the procedure due to advanced pancreatic head neoplasia. One technical failure was experienced (4.3\%). The TTM LAMS placement led to a significant decrease in the serum levels of bilirubin, ALP, GGT, WBC and CRP. No cases of duodenal SEMS occlusion occurred and no other AEs were observed during the follow-up.

Conclusions Concomitant malignant duodenal and biliary obstruction is a challenging condition. Palliation of jaundice using TTM LAMS in patients already treated with duodenal stent is associated to promising technical and clinical outcomes. 


\section{Introduction}

Distal malignant biliary obstruction (MBO) can be caused by different types of tumors, including pancreatic cancer, biliary tract cancer, gallbladder cancer, and metastasis, leading to obstructive jaundice. Endoscopic retrograde cholangiopancreatography (ERCP) is considered the gold standard procedure for jaundice palliation in this setting [1]. However, the presence of surgically altered anatomy (i.e., Whipple intervention, Rouxen-Y gastric bypass, Billroth II surgery), periampullary diverticula, duodenal stent placement, gastric outlet obstruction, and malignant obstruction of the lumen can determine the failure of the procedure in about $5 \%$ to $10 \%$ of cases, requiring alternative methods of biliary decompression [2]. Percutaneous transhepatic biliary drainage (PTBD) and surgical bypass are well established alternatives in these patients, but are also associated with increased morbidity, hospital stay, costs, and patients discomfort $[3,4]$.

In 2001 Giovannini et al. described the first endoscopic ultrasound-guided biliary drainage (EUS-BD) through transduodenal access using a needle knife [5]. Subsequently, EUS-BD has considerably evolved following the development of dedicated devices, such as lumen apposing metal stents (LAMS). LAMS are made of braided nitinol, fully covered with silicone to prevent tissue ingrowth and leakage, and have wide flanges on both ends to provide apposition of the external wall of the two targeted organs and avoid migration of the stents. Currently, three randomized controlled trials (RCTs) comparing EUS-BD vs ERCP have been published, showing similar safety of EUS-BD compared to ERCP, with fewer cases of tumor ingrowth $[6,7]$. Moreover, in one of the studies a longer duration of patency coupled with lower rates of adverse events and reintervention and more preserved QOL were observed in patients undergoing EUS-BD [8].

Recently, LAMS have been incorporated into a delivery system with an electrocautery mounted on the tip (Hot-Axios; Boston Scientific Corp. Marlborough, Massachusetts, United States), which allows the device to be used directly to penetrate the biliary tract without the need of additional devices. In case of malignant jaundice developing after duodenal self-expandable metal stent (SEMS) placement, access to the papilla can be prohibitive or extremely difficult. Thus, the placement of a LAMS through the meshes (TTM) of the duodenal stent can offer an alternative approach to manage malignant jaundice.

The primary aim of our study was to evaluate, in patients with MBO with a previous indwelled duodenal SEMS and in patients with $\mathrm{MBO}$ and gastric outlet obstruction (GOO), the technical feasibility of placement of LAMS TTM of duodenal stents. The secondary aim was to evaluate clinical outcomes and adverse events (AEs) related to the procedures.

\section{Patients and methods}

Data from patients who underwent LAMS placement TTM of a duodenal stent were retrospectively collected in seven referral centers. Inclusion criteria were: age $>18$ years; malignant jaundice with a concomitant gastric outlet obstruction requiring duodenal SEMS placement; malignant jaundice with a previous uncovered duodenal SEMS placed for malignant stricture; patients unfit for surgery; TTM LAMS deployment with or without guidewire placement. Exclusion criteria were: history of PTBD. LAMS were deployed by experienced endoscopists who had performed > 20 LAMS cases for different indications and $>1000$ bilio-pancreatic EUS procedures. Technical success was defined as correct placement of the LAMS through the meshes of a duodenal SEMS. Successful clinical outcome was defined as a bilirubin level decrease $>15 \%$ after 24 hours from the LAMS placement. AEs were divided into early, if they occurred $<24$ hours, or late, if occurred $\geq 24$ hours.

\section{Technical characteristics of the LAMS}

A LAMS is an electrocautery-enhanced fully-covered self-expanding metal stent made of nitinol meshes. The stent is preloaded in a $9 \mathrm{~F}$ or $10.8 \mathrm{~F}$ catheter, with a delivery system compatible with therapeutic echoendoscopes with a working channel of $3.7 \mathrm{~mm}$ diameter or larger. LAMS (Hot Axios-Boston Scientific; Natick, Massachusetts, United States) are currently available with different diameters and lengths: $6 \mathrm{~mm} \times 8 \mathrm{~mm}$, $8 \mathrm{~mm} \times 8 \mathrm{~mm}, 10 \mathrm{~mm} \times 10 \mathrm{~mm}, 15 \mathrm{~mm} \times 10 \mathrm{~mm}$ and $20 \mathrm{~mm} \times$ $10 \mathrm{~mm}$. The $6-\mathrm{mm}$ and $8-\mathrm{mm}$ diameter stents are generally indicated for choledoco-duodenostomy (CDS).

\section{EUS-BD TTM procedure}

All therapeutic EUS with TTM LAMS placement were performed under deep sedation using carbon dioxide $\left(\mathrm{CO}_{2}\right)$ for insufflation. A linear array Olympus and Pentax echoendoscope was used during the procedures. All the procedures were performed in an endoscopy room equipped with X-ray.

When EUS-BD was performed, the common bile duct (CBD) or the gallbladder was identified by EUS from the duodenal bulb, the superior duodenal genu or from the mid descending duodenum. Doppler was used to exclude the presence of interposing vessels. CDS was not attempted in the presence of a CBD diameter $<10 \mathrm{~mm}$, if the distance between the duodenal wall and the $C B D$ was $>10 \mathrm{~mm}$ or in the presence of interposing vessels. The choice to drain the gallbladder or the CBD was at the discretion of the endosonographer, according to the scope stability, position and adequate CBD or gallbladder visualization. The endosonographer choose the easiest access to drain the jaundice, performing a CDS when possible, or a GDS as an alternative if the gallbladder was in situ.

The choice between the TTM LAMS placement technique, with or without previous insertion of a guidewire, and the size of the stent were decided during the procedure by the endosonographers on the basis of their experience.

\section{LAMS placement without wire}

After identifying the target point TTM, the LAMS delivery system was inserted into the working channel of the scope and locked to the inlet port. The delivery was connected to the generator with a pure cut mode. The tip of the LAMS delivery system was then placed tangentially to the bile duct, or the gallbladder, and introduced under EUS guidance into the duct with application of cautery. Once inside the targeted organ, 
the first flange of the LAMS was deployed. Subsequently, the catheter was slightly withdrawn till the appositioning of the distal flange to the wall of the CBD or the gallbladder, then the second flange was deployed, inside the working channel of the echoendoscope using the intra-channel release technique. The echoendoscope was withdrawn while the LAMS outer sheet was advanced releasing the stent out from the operative channel deploying the proximal flange inside the duodenal lumen TTM of the duodenal SEMS.

\section{LAMS placement over the wire}

Once the target point TTM was identified, access to the biliary lumen was obtained using a 19G EUS-needle (Expect 19 G; Boston Scientific,,Natick, Massachusetts, United States) through which a 0.018" guidewire (Novagold; Boston Scientific, Natick, Massachusetts, United States) was advanced into the duct or gallbladder. Subsequently the EUS needle was removed, leaving the guidewire in place, and the LAMS delivery system was mounted over the wire (OTW). After the delivery system was locked to the working channel, the technique was carried out as previously described, removing the guidewire at the end of the procedure.

\section{Statistical considerations}

Continuous data are shown as mean \pm standard deviation (SD) or median and interquartile range (IQR) when not normally distributed. Categorical data are displayed as absolute proportion and percentage (\%). Serum values were reported as T0 before the procedures and as T24 $24 \mathrm{~h}$ after LAMS placement. Tests of hypothesis significance for paired laboratory data, i. e. paired $t$ test or Wilcoxon signed-rank test were provided, as appropriate.

\section{Results}

From February 2016 to April 2020, 23 patients (12 males; mean age: $69.5 \pm 11$ years old) from seven referral endoscopic centers underwent LAMS placement TTM of a duodenal stent for palliation. On the day of the procedure, the mean gastric outlet obstruction score (GOOS) [9] was 2.3 \pm 0.9 ; in particular, four patients $(17.4 \%)$ in which a duodenal SEMS was not in place at time of LAMS deployment had a median GOOS of 1 (IQR 0-1) while the 19 patients (82.6\%) with the duodenal SEMS already indwelled presented with a mean GOOS of $2.72 \pm 0.46$.

In 17 patients (73.9\%), TTM LAMS placement was performed as first intention, whereas in six patients $(26.1 \%)$ it was performed after a failed ERCP attempt. Thirteen patients had an advanced pancreatic head neoplasia (56.5\%) (- Table 1). In four patients (17.4\%) LAMS was placed TTM during the same endoscopic session of the duodenal SEMS deployment ( $\triangleright$ Fig. 1), while in 19 patients (82.6\%) LAMS was placed after a median of 7 days (IQR 5-15; range 2-127 days) from the duodenal stenting. Eleven patients received chemotherapy (CT) (47.8\%) and only three received CT plus radiotherapy (RT) (13\%). After duodenal SEMS placement, all patients were able to restart oral intake the day after the procedure.
- Table 1 Types of neoplasia of the patients who underwent TTM LAMS

\begin{tabular}{|l|c|}
\hline Patients & Number (\%) \\
\hline Pancreatic head adenocarcinoma & $13(56.5 \%)$ \\
\hline Advanced ampulloma & $2(8.7 \%)$ \\
\hline Breast cancer metastases & $2(8.7 \%)$ \\
\hline Distal CBD neoplasia & $2(8.7 \%)$ \\
\hline Pancreatic NET & $1(4.35 \%)$ \\
\hline Duodenal adenocarcinoma & $1(4.35 \%)$ \\
\hline Gallbladder neoplasia & $1(4.35 \%)$ \\
\hline $\begin{array}{l}\text { Recurrence of a previous distal esophageal } \\
\text { adenocarcinoma }\end{array}$ & $1(4.35 \%)$ \\
\hline $\begin{array}{l}\text { TTM, through the mesh; LAMS, lumen apposing metal stent; CBD, common } \\
\text { bile duct; NET, neuroendocrine tumor }\end{array}$ \\
\hline
\end{tabular}

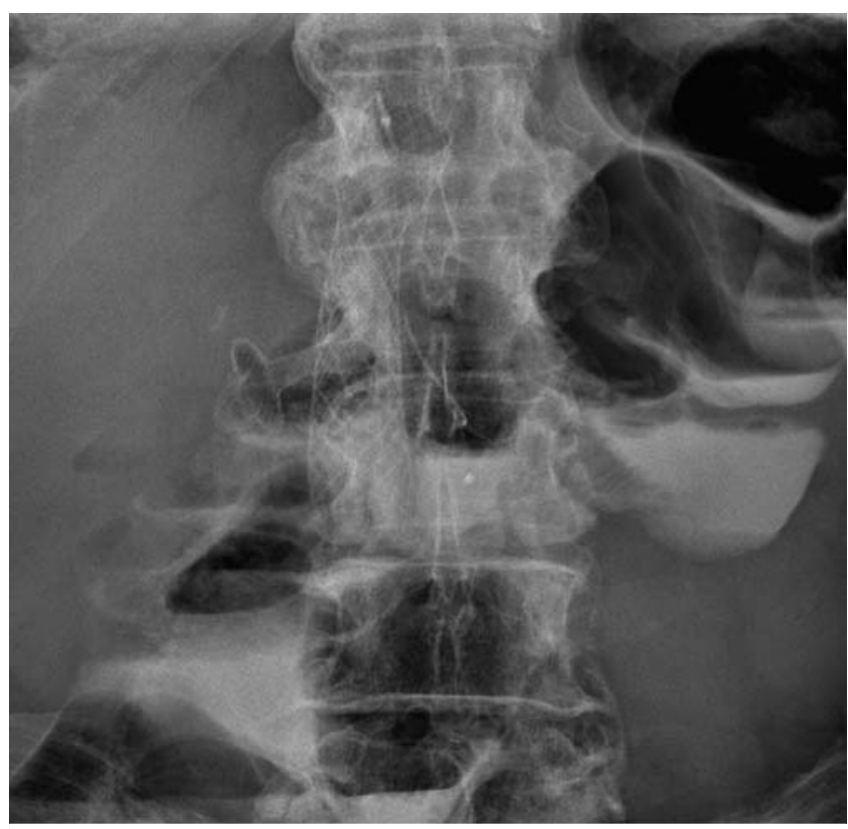

- Fig. 1 X-ray image showing the LAMS inside the mesh of the duodenal SEMS.

LAMS placement led to a significant decrease in serum levels of total and direct bilirubin, alkaline phosphatase, gamma-glutamyl transferase, white blood cells, and C-reactive protein at 24 hours after the procedure ( $\triangleright$ Table 2$)$. All patients were free from jaundice after a median follow-up of 241 days (IQR 81387 days), and no cases of duodenal SEMS occlusion were observed. The major technical features and clinical outcomes for each patient are reported in $>$ Table 3 .

\section{Technical features}

Fourteen patients (60.9\%) underwent gallbladder-duodenostomy (GDS) and nine patients underwent choledoco-duodenostomy (CDS) (39.1\%). Among patients undergoing CDS, the mean diameter of CBD was of $18.3 \pm 6.0 \mathrm{~mm}$. Four procedures 
- Table 2 Decrease in serum levels, in terms of percentage and mean or median value, before and 24 hours after TTM LAMS placement.

\begin{tabular}{|l|c|c|c|c|}
\hline & $\%$ & T0 (serum value) & T24h (serum value) \\
\hline Total bilirubin & $17.1 \%$ & $14.6 \pm 9.3 \mathrm{mg} / \mathrm{dL}$ & $12.1 \pm 8.6 \mathrm{mg} / \mathrm{dL}$ \\
\hline Direct bilirubin & $18.1 \%$ & $10.5 \pm 7.8 \mathrm{mg} / \mathrm{dL}$ & $8.6 \pm 7.2 \mathrm{mg} / \mathrm{dL}$ \\
\hline Aspartate transaminase (AST) & $29.2 \%$ & $133.7 \pm 760.4 \mathrm{U} / \mathrm{L}$ & $94.6 \pm 47.3 \mathrm{U} / \mathrm{L}$ \\
\hline Alanine transaminase (ALT) & $37.1 \%$ & $130.5 \pm 95.5 \mathrm{U} / \mathrm{L}$ & $84 \pm 55.3 / \mathrm{L}$ \\
\hline Alkaline phosphatase (ALP) & $4.8 \%$ & $540(426-1458) \mathrm{U} / \mathrm{L}$ & $514(394-1232) \mathrm{U} / \mathrm{L}$ \\
\hline gamma-glutamyl transferase (YGT) & $6.9 \%$ & $506(300-756) \mathrm{U} / \mathrm{L}$ & $471(225-652) \mathrm{U} / \mathrm{L}$ \\
\hline Amylase & $2.7 \%$ & $123.4 \pm 82.7 \mathrm{U} / \mathrm{L}$ & $120.1 \pm 61.5 \mathrm{U} / \mathrm{L}$ \\
\hline White blood cells (WBC) & $16.9 \%$ & $11.2 \pm 6.1 \times 10^{3} / \mathrm{Ul}$ & 9.015 \\
\hline C-reactive protein (CRP) & $16.3 \%$ & $9.2(7.5-18.5) \mathrm{mg} / \mathrm{dL}$ & $7.7(4.5-12.3) \mathrm{mg} / \mathrm{dL}$ \\
\hline
\end{tabular}

TTM, through the mesh; LAMS, lumen apposing metal stent.

Paired t-test or Wilcoxon signed-rank test were applied, as appropriate.

- Table 3 Technical features and clinical outcomes for each patient.

\begin{tabular}{|c|c|c|c|c|c|}
\hline Patient number & Sex and age & CDS/GDS & оTW & Duodenal stent & Clinical outcome until death \\
\hline 1 & $\mathrm{~F}, 69 \mathrm{yr}$ & CDS & No & Indwelled & No AEs \\
\hline 2 & M, 76 yr & CDS & Yes & Indwelled & Technical failure \\
\hline 3 & M, 77 yr & GDS & No & Placed during the same session & No AEs \\
\hline 4 & M, 74 yr & CDS & No & Indwelled & No AEs \\
\hline 5 & M, $76 \mathrm{yr}$ & CDS & Yes & Placed during the same session & No AEs \\
\hline 6 & $\mathrm{M}, 71 \mathrm{yr}$ & CDS & Yes & Placed during the same session & No AEs \\
\hline 7 & $\mathrm{~F}, 33 \mathrm{yr}$ & GDS & yes & Placed during the same session & No AEs \\
\hline 8 & M, $56 \mathrm{yr}$ & CDS & No & Indwelled & No AEs \\
\hline 9 & M, $79 \mathrm{yr}$ & CDS & No & Indwelled & No AEs \\
\hline 10 & $\mathrm{~F}, 61 \mathrm{yr}$ & GDS & No & Indwelled & No AEs \\
\hline 11 & $\mathrm{~F}, 57 \mathrm{yr}$ & CDS & No & Indwelled & No AEs \\
\hline 12 & $\mathrm{~F}, 78 \mathrm{yr}$ & CDS & No & Indwelled & No AEs \\
\hline 13 & $\mathrm{~F}, 69 \mathrm{yr}$ & GDS & No & Indwelled & No AEs \\
\hline 14 & $\mathrm{~F}, 73 \mathrm{yr}$ & GDS & No & Indwelled & No AEs \\
\hline 15 & $\mathrm{M}, 68 \mathrm{yr}$ & GDS & No & Indwelled & No AEs \\
\hline 16 & $\mathrm{~F}, 86 \mathrm{yr}$ & GDS & No & Indwelled & No AEs \\
\hline 17 & $\mathrm{~F}, 71 \mathrm{yr}$ & GDS & No & Indwelled & No AEs \\
\hline 18 & $\mathrm{M}, 68 \mathrm{yr}$ & GDS & No & Indwelled & No AEs \\
\hline 19 & $\mathrm{~F}, 85 \mathrm{yr}$ & GDS & No & Indwelled & No AEs \\
\hline 20 & $\mathrm{M}, 62 \mathrm{yr}$ & GDS & No & Indwelled & No AEs \\
\hline 21 & $\mathrm{M}, 68 \mathrm{yr}$ & GDS & No & Indwelled & No AEs \\
\hline 22 & $\mathrm{~F}, 69 \mathrm{yr}$ & GDS & No & Indwelled & No AEs \\
\hline 23 & $\mathrm{M}, 74 \mathrm{yr}$ & GDS & No & Indwelled & No AEs \\
\hline
\end{tabular}


(17.4\%) were performed OTW: three CDS and one GDS performed after a transbulbar guide-wire (Novagold; Boston Scientific, Natick, Massachusetts, United States) was released in the CBD using a 19G EUS needle. In all patients except one the LAMS final deployment was performed using the intra-channel release technique (95.6\%) [10]. X-rays were used at the end of all the procedures to check the correct expansion and location of both biliary and duodenal stents. The diameter and length of the duodenal SEMSs were $22 \mathrm{~mm} \times 60 \mathrm{~mm}$ in nine cases (39.1\%), $22 \mathrm{~mm} \times 90 \mathrm{~mm}$ in twelve cases (52.2\%), $22 \times 100 \mathrm{~mm}$ $(4.35 \%)$ in one case, and $22 \times 120 \mathrm{~mm}$ in another case $(4.35 \%)$. All duodenal SEMSs were uncovered (Wallflex; Boston Scientific, Natick, Massachusetts, United States or Niti-S Taewoong; Korea). All LAMSs were Hot-Axios (Boston Scientific, Natick, Massachusetts, United States): $6 \mathrm{~mm} \times 8 \mathrm{~mm}$ in seven cases (30.4\%), $8 \mathrm{~mm} \times 8 \mathrm{~mm}$ in one case $(4.4 \%)$ and $10 \mathrm{~mm} \times 10 \mathrm{~mm}$ in 15 cases $(65.2 \%)$. In all 14 patients who underwent a GDS, a $10 \times 10 \mathrm{~mm}$ LAMS was used. In two patients a biliary uncovered SEMS (8.7\%) had been deployed during a previous procedure, and in another patient an hepatico-gastrostomy (HGS) was in place and occluded. In one patient, due to the presence of cholangitis and cholecystitis, an HGS was also performed during the same session. Technical success was achieved in 22/23 $(95.6 \%)$ patients. In one patient an early migration of the proximal flange out of the CBD into the duodenal lumen occurred immediately after LAMS deployment (4.4\%); thus, the LAMS was immediately removed and the patient underwent a CGS during the same endoscopic procedure. The LAMS was then removed and a trans-gastric drainage of the gallbladder with a second LAMS was performed.

Demographic characteristics of the included patients and the technical aspects of the procedures are reported in $>\mathrm{Ta}$ ble 4 .

\section{Adverse events}

No early or late bleeding, perforation, stent occlusion or acute pancreatitis were encountered. Patients were discharged a median 3 days (IQR 2-3) after the LAMS placement. All patients were followed until death (median survival time from LAMS placement: 241 days, IQR 81-387). No LAMS-related (perforation, occlusion, migration, cholecystitis, cholangitis, bleeding) or duodenal SEMS-related (perforation, occlusion or migration) AEs were recorded.

\section{Discussion}

Ampullary and periampullary malignant diseases, such as pancreatic head neoplasia, distal cholangiocarcinoma, gallbladder neoplasia, or peripancreatic metastatic lesions are usually diagnosed at an advanced stage. Thus, patients are unfit for surgery and only palliative treatment can be accomplished, since CT and RT are no longer indicated for end-stage disease [11]. When a patient is judged unfit for surgery, survival is often less than 6 months and only palliative treatment can be proposed [12]. Concomitant MBO and duodenal obstruction causing gastric outlet obstruction are rare, present in $6 \%$ to $9 \%$ of all periampullary malignancies [13]. Endoscopic duodenal stenting

- Table 4 Demographic characteristics of the patients and technical aspects of the combined procedures.

Number of enrolled patients

Age (years $\pm S D)$

Sex

Previous failed attempt of ERCP

Type of anastomosis

Palliative treatment

LAMS type

LAMStype

Procedure performed after
guide-wire placement
Intrachannel release

Type of duodenal SEMS

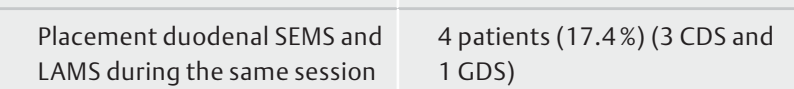

ERCP, endoscopy retrograde cholangiography; SEMS, self expanding metal stent; LAMS, lumen apposing metal stent; CDS, choledoco-duodenostomy; GDS, gallbladder-duodenostomy.

with SEMS insertion is, nowadays, a standardized endoscopic procedure for the relief of the malignant GOO, with a clinical success rate ranging between $84 \%$ to $93 \%$, and a technical success rate ranging between $93 \%$ and $97 \%[14,15]$. Moreover, procedural costs and hospital stay are lower compared to the surgical palliation $[16,17]$.

Endoscopic biliary drainage is currently the most common treatment for jaundice in patients with $\mathrm{MBO}$ mainly by ERCP with stent placement $[18,19]$.

Endoscopic treatment can be challenging when $\mathrm{MBO}$ and duodenal obstruction arise simultaneously or if the MBO occurs subsequent to duodenal SEMS placement. In recent years, LAMS have changed the approach to MBO. EUS-BD is currently performed as a rescue therapy for jaundice palliation after ERCP failure [20]; however, recent RCTs have shown non-superiority of ERCP vs EUS-BD as first intention for biliary drainage [6-8]. The procedure has been described as safe and effective with a low rate of complications [21]. A recent systematic review and meta-analyses showed clinical and technical success rates of EUS-BD of $87 \%$ and $95 \%$, respectively [22].

In our series, no case of recurrent GOO occurred, probably due to the short survival of these patients. In case of a duodenal SEMS occlusion and a TTM LAMS in place, a second duodenal stent can be deployed inside the previous one, ideally not covering the LAMS. If the stenosis occurs at the level of the LAMS, an EUS-guided gastro-jejunostomy [23] should be considered, since the placement of a metal duodenal stent over the LAMS could cause its occlusion. 
Once a duodenal stent is in place, patients with distal MBO have a high-risk of ERCP failure, related to difficulty of CBD cannulation, difficult access to the second part of the duodenum due to SEMS ingrowth, or due to a malignant encasement of the papilla [24]. If the papillary area is reachable, cannulation can still be challenging, implying the need for more advanced cannulation techniques such as pre-cut, double guidewire (DGW) technique or pancreatic septotomy, with a high risk of post-ERCP pancreatitis (PEP) [25].

On the contrary, a recent report did not observe any cases of post-procedural pancreatitis among 46 patients who underwent EUS-BD after failed or unfeasible ERCP [26].

To our knowledge, only two case reports have been published in the literature about placement of a LAMS TTM of an uncovered duodenal stent after failed ERCP $[27,28]$. In our series, we experienced a $95.6 \%$ technical success rate and no early or late AEs were recorded. In particular, no post-procedural pancreatitis was reported. None of the patients experienced abdominal pain immediately or days after TTM LAMS placement. Only one case of technical failure $(4.4 \%)$ occurred, during which complete migration of the LAMS inside the duodenal lumen was observed. The LAMS migration occurred in one of three patients who underwent both $C T$ and $R T$, whereas all other patients underwent only CT or no treatment. Notably, tissue treated by RT often becomes hard and rigid; this could explain the early migration of the LAMS immediately after its deployment [29]. The clinical outcome of the enrolled patients showed a decrease of about one-fifth of the T0 value of the bilirubin and of about one-third of the transaminase value 24 hours after from the procedure. In our cohort, GDS and CDS provided similar outcomes with no significant differences between the two procedures. However, it is still controversial whether GDS should be considered only in case of failure of CDS. Thus, larger studies comparing GDS and CDS in patients with or without duodenal SEMS are warranted to define if the two procedures are equally effective.

Hepaticogastrostomy has been proposed as an alternative procedure for biliary drainage, although it is challenging in expert hands. Thus, in our opinion, with the advent of LAMS, HGS should be limited to cases with malignant hilar obstruction or, more rarely, to patients in which LAMS placement is not feasible due to surgically altered anatomy. Nevertheless, studies comparing these two approaches would provide additional data to define the most appropriate options for palliative jaundice treatment.

\section{Conclusion}

In conclusion, concomitant malignant duodenal and biliary obstructions are rare and challenging conditions for the endoscopist. When a stricture occurs in the papillary area or above it, placement of a duodenal SEMS is mandatory before proceeding to ERCP. After SEMS placement, if ERCP fails, EUS-BD using LAMS can be considered. Draining the CBD or the gallbladder through the duodenal stent mesh is technically feasible and clinically successful. Larger prospective studies are warranted to confirm our promising data.

\section{Competing interests}

The authors declare that they have no conflict of interest.

\section{References}

[1] Dumonceau JM, Tringali A, Papanikolaou IS et al. Endoscopic biliary stenting: indications, choice of stents, and results: European Society of Gastrointestinal Endoscopy (ESGE) Clinical Guideline - Updated October 2017. Endoscopy 2018; 50: 910-930

[2] Enochsson L, Swahn F, Arnelo U et al. Nationwide, population-based data from 11,074 ERCP procedures from the Swedish Registry for Gallstone Surgery and ERCP. Gastrointest Endosc 2010; 72: 11751184

[3] Voegeli DR, Crummy AB, Weese JL. Percutaneous transhepatic cholangiography, drainage, and biopsy in patients with malignant biliary obstruction. An alternative to surgery. Am J Surg 1985; 150: 243-247

[4] Winick AB, Waybill PN, Venbrux AC. Complications of percutaneous transhepatic biliary interventions. Tech Vasc Interv Radiol 2001; 4: 200-206

[5] Giovannini M, Moutardier V, Pesenti C et al. Endoscopic ultrasoundguided bilioduodenal anastomosis: a new technique for biliary drainage. Endoscopy 2001; 33: 898-900

[6] Park Jk, Woo YS, Noh DH et al. Efficacy of EUS-guided and ERCPguided biliary drainage for malignant biliary obstruction: prospective randomized controlled study. Gastrointest Endosco 2018; 88: 277282

[7] Bang JY, Navaneethan U, Hasan M et al. Stent placement by EUS or ERCP for primary biliary decompression in pancreatic cancer: a randomized trial (with videos). Gastrointest Endosc 2018; 88: 9-17

[8] Paik WH, Lee TH, Park DH et al. EUS-Guided Biliary Drainage Versus ERCP for the Primary Palliation of Malignant Biliary Obstruction: A Multicenter Randomized Clinical Trial. Am J Gastroenterol 2018; 113 : 987-997

[9] Adler DG, Baron TH. Endoscopic palliation of malignant gastric outlet obstruction using self-expanding metal stents: experience in 36 patients. Am J Gastroenterol 2002; 97: 72-78

[10] Anderloni A, Attili F, Carrara S et al. Intra-channel stent release technique for fluoroless endoscopic ultrasound-guided lumen-apposing metal stent placement: changing the paradigm. Endosc Int Open 2017; 5: E25-E29

[11] Mangiavillano B, Khashab MA, Tarantino I et al. Success and safety of endoscopic treatments for concomitant biliary and duodenal malignant stenosis: A review of the literature. World J Gastrointest Surg 2019; 11: 53-61

[12] Conio M, Mangiavillano B, Caruso A et al. Covered versus uncovered self-expandable metal stent for palliation of primary malignant extrahepatic biliary strictures: a randomized multicenter study. Gastrointest Endosc 2018; 88: 283-291

[13] Shepherd HA, Royle G, Ross AP et al. Endoscopic biliary endoprosthesis in the palliation of malignant obstruction of the distal common bile duct: a randomized trial. Br J Sur 1988; 75: 1166-1168

[14] van Hooft JE, Uitdehaag MJ, Bruno MJ et al. Efficacy and safety of the new WallFlex enteral stent in palliative treatment of malignant gastric outlet obstruction (DUOFLEX study): a prospective multicenter study. Gastrointest Endosc 2009; 69: 1059-1066

[15] Laasch HU, Martin DF, Maetani I. Enteral stents in the gastric outlet and duodenum. Endoscopy 2005; 37: 74-81

[16] Yoshida Y, Fukutomi A, Tanaka M et al. Gastrojejunostomy versus duodenal stent placement for gastric outlet obstruction in patients 
with unresectable pancreatic cancer. Pancreatology 2017; 17: 983989

[17] Jeurnink SM, Polinder S, Steyerberg EW et al. Cost comparison of gastrojejunostomy versus duodenal stent placement for malignant gastric outlet obstruction. J Gastroenterol 2010; 45: 537-543

[18] Mangiavillano B, Montale A, Frazzoni L et al. Endoscopic biliary selfexpandable metallic stent in malignant biliary obstruction with or without sphincterotomy: systematic review and meta-analysis. Endosc Int Open 2019; 7: E26-E35

[19] Mangiavillano B, Papanikolaou IS, Arvanitakis M et al. Endoscopic drainage in patients with malignant extrahepatic biliary obstruction: when and how. Eur J Gastroenterol Hepatol 2020: doi:10.1097| MEG.0000000000001752 [Epub ahead of print]

[20] Guo ], Giovannini M, Sahai AV et al. A multi-institution consensus on how to perform EUS-guided biliary drainage for malignant biliary obstruction. Endosc Ultrasound 2018; 7: 356-365

[21] Kunda R, Perez-Miranda M, Will U et al. EUS-guided choledochoduodenostomy for malignant distal biliary obstruction using a lumen-apposing fully covered metal stent after failed ERCP. Surg Endosc 2016; 30: 5002-5008

[22] Hedjoudje A, Sportes A, Grabar S et al. Outcomes of endoscopic ultrasound-guided biliary drainage: A systematic review and meta-analysis. United European Gastroenterol J 2019; 7: 60-68
[23] Wannhoff A, Ruh N, Meier B et al. Endoscopic gastrointestinal anastomoses with lumen-apposing metal stents: predictors of technical success. Surg End 2020: doi:10.1007/S00464-020-07594-5

[24] Yao JF, Zhang L, Wu H. Analysis of high risk factors for endoscopic retrograde cholangiopancreatography biliary metallic stenting after malignant duodenal stricture SEMS implantation. J Biol Regul Homeost Agents 2016; 30: 743-748

[25] Pereira Lima JC, Arciniegas Sanmartin ID, Latrônico Palma B et al. Risk factors for success, complications and death after endoscopic sphincterotomy for bile duct stones: a 17-year experience with 2137 cases. Dig Dis 2020; 38: 534-541

[26] Anderloni A, Fugazza A, Troncone E et al. Single-stage EUS-guided choledochoduodenostomy using a lumen-apposing metal stent for malignant distal biliary obstruction. Gastrointest Endosc 2019; 89: 69-76

[27] Sportes A, Airinei G, Kamel R et al. Endoscopic ultrasound-guided choledochoduodenostomy with a lumen-apposing metal stent through an uncovered metal duodenal stent. Endosc Int Open 2018; 6: E1395-1397

[28] Anderloni A, Fugazza A, Auriemma F et al. cautery-tipped lumen apposing metal stent placement through the mesh of an indwelling duodenal self-expanding metal stent. Am J Gastroenterol 2018; 113: 644

[29] Seifert WF, Wobbes T, Hoogenhout J et al. Intraoperative irradiation delays anastomotic repair in rat colon. Am J Surg 1995; 170: 256-261 